\title{
THE EXTENDED $\lambda$-METHOD FOR CONTROLLED LAGRANGIAN SYSTEMS
}

\author{
Dong Eui Chang
}

Institute of Montefiore, University of Liège, Belgium.

\begin{abstract}
This paper extends the $\lambda$-method, which was developed in (Auckly et al., 2000; Auckly and Kapitanski, 2003) to solve effectively PDE's involved in the method of controlled Lagrangian systems, by taking into account a gyroscopic term. The gyroscopic force provides more free parameters when one designs stabilizing controllers in the method of controlled Lagrangian systems. We illustrate the extended $\lambda$-method in the example of the Furuta pendulum. Copyright $^{\circledR}$ 2005 IFAC.
\end{abstract}

Keywords: Stabilization methods, mechanical systems

\section{INTRODUCTION}

The control of mechanical systems is a big branch in the area of control theory and application. The method of controlled Lagrangian (CL) systems has been developed to help design stabilizing controllers for mechanical systems (Bloch et al., 1997; Bloch et al., 2000; Bloch et al., 2001; Chang et al., 2002; Chang and Marsden, 2004; Hamberg, 2000; Auckly et al., 2000; Auckly and Kapitanski, 2003). In parallel, there has been a development of its Hamiltonian counterpart, which is called port-controlled Hamiltonians (van der Schaft, 2000; Blankenstein et al., 2002; Ortega et al., 2002) or controlled Hamiltonian (CH) systems (Chang et al., 2002). The relation between these two methods - Lagrangian and Hamiltonian - was studied by (Blankenstein et al., 2002; Chang et al., 2002). The latter showed the equivalence of the two methods for simple mechanical control systems. It also generalized the theory of CL systems taking into account external forces (Chang et al., 2002). This external force can be used not only to take into account all external forces but also to help design stabilizing controllers with an additional gyroscopic force, which is related to the failure of the Jacobi identity of a Poisson tensor on the Hamiltonian side (Chang et al., 2002).
The method of CL systems dictates so called matching conditions, which involve a system of first order quasi-linear PDE's. To effectively solve the PDE's, the $\lambda$-method was developed (Auckly et al., 2000) which splits the PDE's into two systems of first-order linear PDE's. The purpose of the current paper is to extend the $\lambda$-method so that it takes into account the generalization of the controlled Lagrangian systems made in (Chang et al., 2002). A similar work was done in a coordinate-dependent language during the process of transforming the matching conditions on the Hamiltonian side to the Lagrangian side (Blankenstein et al., 2002). In this paper, we make a complete development of the extended $\lambda$-method in a coordinate-free way exclusively on the Lagrangian side. The work in this paper is done mutatis mutandis following (Auckly et al., 2000; Auckly and Kapitanski, 2003).

\section{MAIN RESULTS}

\subsection{Review of the method of CL systems}

We briefly review the method of controlled Lagrangian systems (Chang, 2002; Chang et al., 2002). 
Definition 1. A (simple) controlled Lagrangian system is a triple, $(L, F, W)$ where $L(q, \dot{q})=$ $\frac{1}{2} m_{q}(\dot{q}, \dot{q})-V(q)$ is a (simple) Lagrangian of the kinetic minus potential energy form with $m \in$ $\Gamma\left(T^{*} Q \otimes T^{*} Q\right)$ nondegenerate, $F: T Q \rightarrow T^{*} Q$ is an external force, and $W$ is a subbundle of $T^{*} Q$ called control subbundle.

Feedback controllers are fiber-preserving maps of $T Q \rightarrow W \subset T^{*} Q$. The equations of motion of the system $(L, F, W)$ with a choice of a controller $u$ is given by

$$
\mathcal{E} \mathcal{L}(L)=F+u
$$

where $\mathcal{E} \mathcal{L}$ is the Euler-Lagrange operator defined by

$$
\mathcal{E} \mathcal{L}(L):=\frac{d}{d t} \frac{\partial L}{\partial \dot{q}}-\frac{\partial L}{\partial q} .
$$

Once we choose a control law $u$, then the triple $(L, F, u)$ denotes the closed-loop system.

Definition 2. Given two systems $\left(L_{1}, F_{1}, W_{1}\right)$ and $\left(L_{2}, F_{2}, W_{2}\right)$, the Euler-Lagrange matching conditions are

ELM-1 : $W_{1}=m_{1} m_{2}^{-1}\left(W_{2}\right)$,

ELM-2 : $\operatorname{Im}\left[\left(\mathcal{E} \mathcal{L}\left(L_{1}\right)-F_{1}\right)-m_{1} m_{2}^{-1}\left(\mathcal{E} \mathcal{L}\left(L_{2}\right)-\right.\right.$ $\left.\left.F_{2}\right)\right] \subset W_{1}$,

where Im means the pointwise image of the linear map in brackets. We say that the two simple Lagrangian systems $\left(L_{1}, F_{1}, W_{1}\right)$ and $\left(L_{2}, F_{2}, W_{2}\right)$ are equivalent if ELM-1 and ELM-2 hold. We use the symbol $\stackrel{L}{\sim}$ for this equivalence relation.

The following proposition is from (Chang et al., 2002).

Proposition 3. Suppose two simple controlled Lagrangian systems $\left(L_{1}, F_{1}, W_{1}\right)$ and $\left(L_{2}, F_{2}, W_{2}\right)$ are equivalent. Then, for any controller $u_{2}: T Q \rightarrow$ $W_{2}$ there exists a controller $u_{1}: T Q \rightarrow W_{1}$ such that the two closed-loop system $\left(L_{1}, F_{1}, u_{1}\right)$ and $\left(L_{2}, F_{2}, u_{2}\right)$ produce the same equations of motion, and vice versa. The explicit relation between $u_{1}$ and $u_{2}$ is given by

$$
\begin{aligned}
u_{1}= & \left(\mathcal{E} \mathcal{L}\left(L_{1}\right)-F_{1}\right)-m_{1} m_{2}^{-1}\left(\mathcal{E} \mathcal{L}\left(L_{2}\right)-F_{2}\right) \\
& +m_{1} m_{2}^{-1} u_{2}
\end{aligned}
$$

where $m_{i}$ is the mass matrix of the Lagrangian $L_{i}$ with $i=1,2$.

\subsection{Extended $\lambda$-Method}

We extend the $\lambda$-method, which was introduced in (Auckly et al., 2000), by considering the full form of simple CL system $(L, F, W)$ defined in Definition 1.
First, we review how to get equations of motion of a CL system $(L, F, W)$ using the Levi-Civita connection. It is well known that the equation of motion (1) of a CL system $(L, F, W)$ with

$$
L(q, \dot{q})=\frac{1}{2} m(q)(\dot{q}, \dot{q})-V(q)
$$

can be written on $T Q$ as follows:

$$
\nabla_{\dot{q}} \dot{q}+m^{-1} \mathbf{d} V=m^{-1} F+m^{-1} u,
$$

where $\nabla$ is the Levi-Civita connection of the metric $m$. Let $P \in \Gamma\left(T^{*} Q \otimes T Q\right)$ be the $m$ orthogonal projection with ker $P=m^{-1} W$ where the $m$-orthogonality means

$$
m(P X, Y)=m(X, P Y) .
$$

As the projection $P$ and the control subbundle $W$ have the same information, we will equivalently use $(L, F, W)$ or $(L, F, P)$ depending on the context.

We now reformulate the Euler-Lagrange matching conditions in Definition 2 with the Levi-Civita connection. Consider two CL systems $\left(L_{i}, F_{i}, W_{i}\right)$, $i=1,2$ with

$$
L_{i}(q, \dot{q})=\frac{1}{2} m_{i}(q)(\dot{q}, \dot{q})-V_{i}(q)
$$

Let $\nabla^{i}$ be the Levi-Civita connection of the metric $m_{i}$, and $P_{i} \in \Gamma\left(T^{*} Q \otimes T Q\right)$ the $m_{i}$-orthogonal projection with $\operatorname{ker} P_{i}=m_{i}^{-1} W_{i}$. Then, the Euler-Lagrange matching conditions, ELM-1 and ELM-2 in Definition 2 can be equivalently written as follows:

$$
\begin{aligned}
& \text { ELM-1 }^{\prime}: \quad \operatorname{ker} P_{1}=\operatorname{ker} P_{2} \\
& \text { ELM-2 }^{\prime}: \quad P_{1}\left[\nabla_{\dot{q}}^{1} \dot{q}-\nabla_{\dot{q}}^{2} \dot{q}+m_{1}^{-1} \mathbf{d} V_{1}-m_{2}^{-1} \mathbf{d} V_{2}-\right. \\
& \left.\quad\left(m_{1}^{-1} F_{1}-m_{2}^{-1} F_{2}\right)\right]=0 .
\end{aligned}
$$

We are interested in the following question:

Given a $C L$ system $\left(L_{1}, F_{1}, W_{1}\right)$ (or, equivalently $\left.\left(L_{1}, F_{1}, P_{1}\right)\right)$, find its $C L$-equivalent $C L$ systems.

One can regard ELM-1 and ELM-2 (or, equivalently, ELM-1' and ELM-2' ${ }^{\prime}$ ) as partial differential equations for $\left(L_{2}, F_{2}, W_{2}\right)$ (or, $\left(L_{2}, F_{2}, P_{2}\right)$ ). Without loss of generality, one may assume that $F_{1}=0$ by letting

$$
F_{2} \mapsto F_{2}+m_{2} m_{1}^{-1} F_{1}
$$

We decompose $F_{2}$ as follows:

$$
F_{2}(q, \dot{q})=F_{2}^{q}(q)+F_{2}^{v}(q, \dot{q}),
$$

where $F_{2}^{q}(q)$ contains all the terms in $F_{2}$ which do not depend on the velocity $\dot{q}$. Then, by collecting the terms dependent on $\dot{q}$ and those independent of $\dot{q}, \mathbf{E L M - 2} \mathbf{2}^{\prime}$ is split into the two conditions:

$$
\begin{aligned}
& P_{1}\left(\nabla_{X}^{1} X-\nabla_{X}^{2} X+m_{2}^{-1} F_{2}^{v}(X)\right)=0, \\
& P_{1}\left(m_{1}^{-1} \mathbf{d} V_{1}-m_{2}^{-1} \mathbf{d} V_{2}+m_{2}^{-1} F_{2}^{q}\right)=0
\end{aligned}
$$

for $X \in T Q$. 
Let $\lambda=m_{2}^{-1} m_{1} \in \Gamma\left(T^{*} Q \otimes T Q\right)$. This operator $\lambda$ was introduced in (Auckly et al., 2000). Then $\lambda$ is $m_{1}$ self-adjoint, i.e., $m_{1} \lambda=\lambda^{*} m_{1} \in \Gamma\left(T^{*} Q \otimes\right.$ $\left.T^{*} Q\right)$, or

$$
m_{1}(\lambda X, Y)=m_{1}(X, \lambda Y)
$$

for $X, Y \in T Q$.

Propositions 4, 5, 6 and 7 in the following are the extension of propositions in (Auckly et al., 2000) by taking into account the terms $F_{2}^{q}$ and $F_{2}^{v}$. The proofs can be done mutatis mutandis.

Proposition 4. Let $\lambda=m_{2}^{-1} m_{1}$ and assume that $m_{2}$ and $F_{2}^{v}$ satisfy (5). Then, $\lambda$ satisfies

$0=\nabla_{Z}^{1}\left(m_{1} \lambda\right)(P X, P X)$

$+\left\langle F_{2}^{v}\left(\lambda P_{1} X+Z\right)-F_{2}^{v}\left(\lambda P_{1} X\right)-F_{2}^{v}(Z), \lambda P_{1} X\right\rangle$

for $X, Z \in T Q$.

Proposition 5. Let $\lambda=m_{2}^{-1} m_{1}$, and assume $m_{2}$ and $F_{2}^{v}$ satisfy (5). Then, $m_{2}$ satisfies

$$
\begin{aligned}
& L_{\lambda P_{1} X} m_{2}(Z, Z) \\
& \quad=L_{P_{1} X} m_{1}(Z, Z)-2\left\langle F_{2}^{v}(Z), \lambda P_{1} X\right\rangle
\end{aligned}
$$

for $X, Z \in T Q$.

Proposition 6. The condition (6) for $V_{2}$ is the same as the following:

$$
L_{\lambda P_{1} X} V_{2}=L_{P_{1} X} V_{1}+\left\langle F_{2}^{q}, \lambda P_{1} X\right\rangle
$$

for $X \in T Q$.

A brief explanation of Propositions 4 and 5 is in order. Propositions 4 and 5 convert the firstorder quasi-linear PDE of $m_{2}$ into a first-order quasi-linear PDE (8) for $\lambda$ and a first-order linear PDE (9) for $m_{2}$. Although (8) is quasi-linear, one can notice that the coefficients of the derivatives of $\lambda$ do not depend on $\lambda$. Hence, this splitting makes it easy to solve (5) for $m_{2}$. Notice that we are free to choose $F_{2}^{v}$ in (8) and (9), which allows more solutions for $m_{2}$ than the original $\lambda$ method in (Auckly et al., 2000). We will make use of this additional term $F_{2}^{v}$ to design a stabilizing controller in $\S 2.4$.

The following proposition summarizes the extended $\lambda$-method:

Proposition 7. Let $\lambda=m_{2}^{-1} m_{1}$. Then, $m_{2}$ and $F_{2}^{v}$ satisfy (5) if and only if $\lambda, F_{2}^{v}$ and $m_{2}$ satisfy (8) and (9). The equation (6) is equivalent to the following: for $X \in T Q$

$$
L_{\lambda P_{1} X} V_{2}=L_{P_{1} X} V_{1}+\left\langle F_{2}^{q}, \lambda P_{1} X\right\rangle .
$$

Once $m_{2}$ is derived, $W_{2}$ is given by $W_{2}=$ $m_{2} m_{1}^{-1} W_{1}$.

\subsection{Application to Stabilization Problems}

We apply the extended $\lambda$-method to stabilization problems. Here, we derive general formulae and in $\S 2.4$ we apply them to the problem of stabilization of the inverted pendulum on a rotor arm (the Furuta pendulum).

In this section we keep the notations used in (Auckly and Kapitanski, 2003) as the result in this section is a direct extension of the original work in (Auckly and Kapitanski, 2003).

Denote by $Q$ the configuration space of dimension $s$. Suppose that we are given a CL system $\left(L_{1}, F_{1}=0, W_{1}\right)=(L, 0, W)$ with

$$
L(q, \dot{q})=\frac{1}{2} \sum_{i=1}^{s} m_{i j}(q) \dot{q}^{i} \dot{q}^{j}-V(q)
$$

and

$$
W=\left\langle\mathbf{d} q^{a} \mid a=r+1, \ldots, s\right\rangle
$$

with $r<s$. In this section, we use indices as follows:

$$
\begin{aligned}
& i, j, k, l=1, \ldots, s, \\
& \alpha, \beta, \gamma=1, \ldots, r(<s), \\
& a, b, c=r+1, \ldots, s .
\end{aligned}
$$

The $m$-orthogonal projection $P \in \Gamma\left(T^{*} Q \otimes T Q\right)$ with ker $P=m^{-1} W$ is given in coordinates by

$$
\left\{\begin{array}{l}
P\left(\frac{\partial}{\partial q^{\alpha}}\right)=\frac{\partial}{\partial q^{\alpha}} \text { if } \alpha=1, \ldots, r \\
P\left(m^{i a} \frac{\partial}{\partial q^{i}}\right)=0 \text { if } a=r+1, \ldots, s .
\end{array}\right.
$$

We want to find CL systems $\left(L_{2}, F_{2}, W_{2}\right)=$ $(\widehat{L}, \widehat{F}, \widehat{W})$ with $\widehat{F}$ gyroscopic force quadratic in $\dot{q}$ of the following form:

$$
\widehat{L}(q, \dot{q})=\frac{1}{2} \widehat{m}_{i j}(q) \dot{q}^{i} \dot{q}^{j}-\widehat{V}(q)
$$

and

$$
\begin{aligned}
\widehat{F}(X) & =\widehat{F}(X)_{k} \mathbf{d} q^{k}=\widehat{G}_{i j k}(q) X^{i} X^{j} \mathbf{d} q^{k}, \\
\widehat{G}_{i j k} & =-\widehat{G}_{i k j}
\end{aligned}
$$

with

$\widehat{G}=\widehat{G}_{i j k} \mathbf{d} q^{i} \otimes \mathbf{d} q^{j} \otimes \mathbf{d} q^{k} \in \Gamma\left(T^{*} Q \otimes T^{*} Q \otimes T^{*} Q\right)$, where the skew symmetry in the last two indices of $G_{i j k}$ comes from the fact that $\widehat{F}$ is gyroscopic. Among these equivalent systems, we will use a CL system whose energy has a minimum at the equilibrium of interest in applications.

We apply Propositions $4-7$ to the current problem, and then express the extended $\lambda$-method in coordinates.

Proposition 8. Suppose $(L, 0, W) \stackrel{L}{\sim}(\widehat{L}, \widehat{F}, \widehat{W})$, where

$$
\begin{aligned}
& L=\frac{1}{2} m(\dot{q}, \dot{q})-V(q), \quad \widehat{L}=\frac{1}{2} \widehat{m}(\dot{q}, \dot{q})-\widehat{V}(q), \\
& W=\left\langle\mathbf{d} q^{a} \mid a=r+1, \ldots, s\right\rangle, \quad \widehat{F}(X)=\widehat{G}(X, X)
\end{aligned}
$$


for $X \in T Q$ with $\operatorname{dim} Q=s$ and $\widehat{G}$ a $(0,3)$-tensor satisfying $\widehat{G}_{i j k}=-\widehat{G}_{i k j}$. Let $\nabla$ be the Levi-Civita connection of the metric $m$, and $P$ be the $m$ orthogonal projection with ker $=m^{-1} W$. Then, the following holds: for all $X, Y, Z \in T Q$

$$
\begin{aligned}
& \nabla_{Z}(m \lambda)(P X, P Y) \\
& =\frac{1}{2}\langle\widehat{G}(\lambda P X, \lambda P Y)-\widehat{G}(\lambda P Y, \lambda P X), Z\rangle \\
& L_{\lambda P X} \widehat{m}(Y, Z) \\
& =L_{P X} m(Y, Z)-\langle\widehat{G}(Y, Z)+\widehat{G}(Z, Y), \lambda P X\rangle \\
& L_{\lambda P X} \widehat{V}=L_{P X} V .
\end{aligned}
$$

In coordinates,

$$
\begin{gathered}
\frac{\partial\left(m_{\alpha i} \lambda_{\beta}^{i}\right)}{\partial q^{k}}-[\alpha k, i] \lambda_{\beta}^{i}-[\beta k, i] \lambda_{\alpha}^{i} \\
=\frac{1}{2}\left(\widehat{G}_{i j k}+\widehat{G}_{j i k}\right) \lambda_{\alpha}^{i} \lambda_{\beta}^{j}, \\
\lambda_{\alpha}^{k} \frac{\partial \widehat{m}_{i j}}{\partial q^{k}}+\frac{\partial \lambda_{\alpha}^{k}}{\partial q^{i}} \widehat{m}_{k j}+\frac{\partial \lambda_{\alpha}^{k}}{\partial q^{j}} \widehat{m}_{k i} \\
=\frac{\partial m_{i j}}{\partial q^{\alpha}}-\left(\widehat{G}_{i j k}+\widehat{G}_{j i k}\right) \lambda_{\alpha}^{k}, \\
\lambda_{\alpha}^{k} \frac{\partial \widehat{V}}{\partial q^{k}}=\frac{\partial V}{\partial q^{\alpha}}
\end{gathered}
$$

where $i, j, k=1, \ldots, s$, and $\alpha, \beta=1, \ldots, r$.

We remark that one can recover the results in (Auckly and Kapitanski, 2003) if $\widehat{G}_{i j k}$ is set to zero.

\subsection{Example: Inverted Pendulum on a Rotor Arm}

We apply the extended $\lambda$-method to the design of an asymptotically stabilizing feedback control law for the inverted pendulum on a rotor arm shown in Figure 1. With the method of CL systems a controller was designed to asymptotically stabilize the vertical position of the arm and put the lower arm to rest in (Bloch et al., 1999). We here asymptotically stabilize the position of the lower arm as well at a designed point.
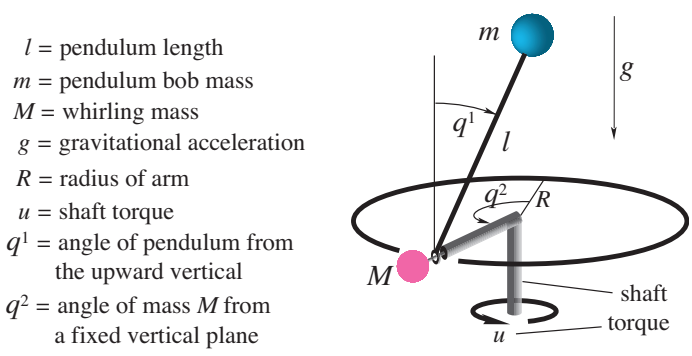

Fig. 1. Inverted pendulum on a rotor arm.

The configuration space is $Q=S^{1} \times S^{1}$. We will use $\left(q^{1}, q^{2}\right)$ as local coordinates where $q^{1}$ is the angle of the pendulum from the upward vertical and $q^{2}$ is the angle of mass $M$ from a fixed vertical plane. This system can be described as a CL system $(L, 0, W)$ with

$$
\begin{aligned}
L\left(q^{1},\right. & \left.q^{2}, \dot{q}^{2}, \dot{q}^{2}\right) \\
& =\frac{1}{2} m l^{2}\left(\dot{q}^{1}\right)^{2}+m R l \cos \left(q^{1}\right) \dot{q}^{1} \dot{q}^{2} \\
& +\frac{1}{2}\left((m+M) R^{2}+m l^{2} \sin ^{2}\left(q^{1}\right)\right)\left(\dot{q}^{2}\right)^{2} \\
& -m g l \cos \left(q^{1}\right),
\end{aligned}
$$

and

$$
W=\left\langle\mathbf{d} q^{2}\right\rangle .
$$

For simplicity, we will use the following Lagrangian instead:

$$
\begin{aligned}
& L\left(q^{1}, q^{2}, \dot{q}^{2}, \dot{q}^{2}\right) \\
& =\frac{1}{2}\left(\dot{q}^{1}\right)^{2}+\cos \left(q^{1}\right) \dot{q}^{1} \dot{q}^{2}+\frac{1}{2}\left(2+\sin ^{2}\left(q^{1}\right)\right)\left(\dot{q}^{2}\right)^{2} \\
& \quad-\cos \left(q^{1}\right) .
\end{aligned}
$$

Then, the nonzero Christoffel symbols of the first kind (Spivak, 1979) are given by

$$
\begin{aligned}
& {[11,2]=-\sin \left(q^{1}\right),} \\
& {[12,2]=[21,2]=\sin \left(q^{1}\right) \cos \left(q^{1}\right),} \\
& {[22,1]=-\sin \left(q^{1}\right) \cos \left(q^{1}\right) .}
\end{aligned}
$$

The $\lambda$-equations in (16) are given by

$$
\left\{\begin{aligned}
& \frac{\partial \lambda_{1}^{1}}{\partial q^{1}}+\cos \left(q^{1}\right) \frac{\partial \lambda_{1}^{2}}{\partial q^{1}}+\sin \left(q^{1}\right) \lambda_{1}^{2} \\
&=\lambda_{1}^{1} \lambda_{1}^{2} \widehat{G}_{121}+\lambda_{1}^{2} \lambda_{1}^{2} \widehat{G}_{221}, \\
& \frac{\partial \lambda_{1}^{1}}{\partial q^{2}}+\cos \left(q^{1}\right) \frac{\partial \lambda_{1}^{2}}{\partial q^{2}}-2 \sin \left(q^{1}\right) \cos \left(q^{1}\right) \lambda_{1}^{2} \\
&=\lambda_{1}^{1} \lambda_{1}^{1} \widehat{G}_{112}+\lambda_{1}^{1} \lambda_{1}^{2} \widehat{G}_{212} .
\end{aligned}\right.
$$

In stead of dealing with general solutions of (19), we will restrict ourselves to a particular solution. To simplify the second equation in (19), we choose

$$
\begin{aligned}
& \widehat{G}_{212}=-\widehat{G}_{221}=-\frac{1}{\lambda_{1}^{1}} 2 \sin \left(q^{1}\right) \cos \left(q^{1}\right), \\
& \widehat{G}_{112}=\widehat{G}_{121}=0 .
\end{aligned}
$$

Then, (19) becomes

$$
\left\{\begin{array}{l}
\frac{\partial \lambda_{1}^{1}}{\partial q^{1}}+\cos \left(q^{1}\right) \frac{\partial \lambda_{1}^{2}}{\partial q^{1}}+\sin \left(q^{1}\right) \lambda_{1}^{2} \\
=\frac{\lambda_{1}^{2} \lambda_{1}^{2}}{\lambda_{1}^{1}} 2 \sin \left(q^{1}\right) \cos \left(q^{1}\right), \\
\frac{\partial \lambda_{1}^{1}}{\partial q^{2}}+\cos \left(q^{1}\right) \frac{\partial \lambda_{1}^{2}}{\partial q^{2}}=0 .
\end{array}\right.
$$

We will use the following particular solution to (20):

$$
\lambda_{1}^{1}=-\frac{1}{k_{1}}, \quad \lambda_{1}^{2}=\frac{\cos \left(q^{1}\right)}{k_{2}-k_{1} \cos ^{2}\left(q^{1}\right)}
$$

with $k_{1}, k_{2} \in \mathbb{R}$. Hence,

$$
\begin{aligned}
& \widehat{G}_{212}=-\widehat{G}_{221}=2 k_{1} \sin \left(q^{1}\right) \cos \left(q^{1}\right), \\
& \widehat{G}_{112}=\widehat{G}_{121}=0 .
\end{aligned}
$$


With this solution, equations (17) become

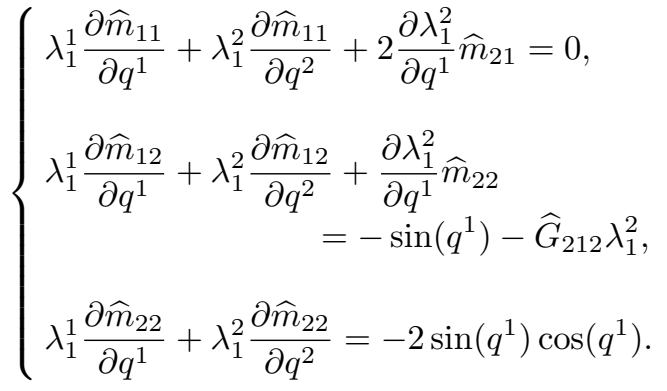

One can solve the third equation in (21) for $\widehat{m}_{22}$ as follows:

$$
\widehat{m}_{22}\left(q^{1}, q^{2}\right)=c_{22}\left(x^{2}\left(q^{1}, q^{2}\right)\right)-k_{1} \cos ^{2}\left(q^{1}\right),
$$

where $c_{22}$ is an arbitrary function of $x^{2}$ and $x^{2}$ is defined by

$$
\begin{aligned}
& x^{2}\left(q^{1}, q^{2}\right):=q^{2} \\
& +\sqrt{\frac{k_{1}}{k_{2}-k_{1}}} \arctan \left(\frac{2 \tan \left(\frac{q^{1}}{2}\right)}{1+\tan ^{2}\left(\frac{q^{1}}{2}\right)} \sqrt{\frac{k_{1}}{k_{2}-k_{1}}}\right) .
\end{aligned}
$$

Now, one does not need to solve the first two equations in (21) for $\widehat{m}_{12}, \widehat{m}_{11}$. Using the relation $\widehat{m} \lambda=m$, one gets

$$
\begin{aligned}
& \widehat{m}_{12}=\frac{-k_{1}\left(k_{2}-c_{22}\left(x^{2}\right)\right) \cos \left(q^{1}\right)}{k_{2}-k_{1} \cos ^{2}\left(q^{1}\right)}, \\
& \widehat{m}_{11}=-k_{1}\left(1+\frac{k_{1}\left(k_{2}-c_{22}\left(x^{2}\right)\right) \cos ^{2}\left(q^{1}\right)}{\left(k_{2}-k_{1} \cos ^{2}\left(q^{1}\right)\right)^{2}}\right) .
\end{aligned}
$$

The equation (18) is given by

$$
\lambda_{1}^{1} \frac{\partial \widehat{V}}{\partial q^{1}}+\lambda_{1}^{2} \frac{\partial \widehat{V}}{\partial q^{2}}=-\sin \left(q^{1}\right)
$$

whose solution is

$$
\widehat{V}\left(q^{1}, q^{2}\right)=-k_{1} \cos ^{2}\left(q^{1}\right)+U\left(x^{2}\left(q^{1}, q^{2}\right)\right)
$$

where $U(\cdot)$ is an arbitrary function. The control bundle $\widehat{W}=\widehat{m} m^{-1} W$ is given by

$$
\widehat{W}=\left\langle\mathbf{d} q^{2}+\frac{k_{1} \cos \left(q^{1}\right)}{k_{2}-k_{1} \cos ^{2}\left(q^{1}\right)} \mathbf{d} q^{1}\right\rangle .
$$

So far, we have found a CL system $(\widehat{L}, \widehat{F}, \widehat{W})$ parameterized by real numbers $k_{1}, k_{2}$ and functions $c_{22}, U$, which is equivalent to the original system. Hence, we will equivalently work with $(\widehat{L}, \widehat{F}, \widehat{W})$ to find a controller that asymptotically stabilizes $(0,0,0,0)$. To simplify computation, we use the new coordinates $\left(x^{1}=q^{1}, x^{2}\right)$ with $x^{2}$ in (22). In the new coordinates $\left(x^{1}, x^{2}\right)$, the CL system $(\widehat{L}, \widehat{F}, \widehat{W})$ is expressed as

$$
\begin{aligned}
& \widehat{m}_{x}=\left[\begin{array}{cc}
\frac{-k_{1}\left(k_{2}-2 k_{1} \cos ^{2}\left(x^{1}\right)\right)}{k_{2}-k_{1} \cos ^{2}\left(x^{1}\right)} & -k_{1} \cos \left(x^{1}\right) \\
-k_{1} \cos \left(x^{1}\right) & c_{22}\left(x^{2}\right)-k_{1} \cos ^{2}\left(x^{1}\right)
\end{array}\right], \\
& \widehat{V}(x)=-k_{1} \cos \left(x^{1}\right)+U\left(x^{2}\right), \\
& \widehat{F}(x, \dot{x})=k_{1} \sin \left(2 x^{1}\right)\left(\dot{x}^{2}-\frac{k_{1} \cos \left(x^{1}\right) \dot{x}^{1}}{k_{2}-k_{1} \cos ^{2}\left(x^{1}\right)}\right) \\
& \quad \times\left(-\dot{x}^{2} \mathbf{d} x^{1}+\dot{x}^{1} \mathbf{d} x^{2}\right), \\
& \widehat{W}=\left\langle\mathbf{d} x^{2}\right\rangle .
\end{aligned}
$$

Let $\widehat{E}=\frac{1}{2} \widehat{m}(\dot{x}, \dot{x})+\widehat{V}(x)$ be the energy of the CL system $(\widehat{L}, \widehat{F}, \widehat{W})$. One can check that the energy $\widehat{E}$ has a local minimum at $(0,0,0,0)$ if

$$
\begin{aligned}
& c_{22}(0)>k_{1}>0, \\
& \frac{k_{1}\left(k_{1}^{2}-2 k_{1} c_{22}(0)+k_{2} c_{22}(0)\right)}{-k_{2}+k_{1}}>0, \\
& U^{\prime}(0)=0, \quad U^{\prime \prime}(0)>0 .
\end{aligned}
$$

To make the new coordinates $\left(x^{1}, x^{2}\right)$ be realvalued coordinates, we need the following additional condition:

$$
k_{2}>k_{1} \text {. }
$$

One can always find some parameters $k_{1}, k_{2}$, $c_{22}\left(x^{2}\right)$, and $U\left(x^{2}\right)$ satisfying $(23)-(26)$. Here, for simplicity, we use the following $U$ :

$$
U\left(x^{2}\right)=\frac{1}{2} \epsilon\left(x^{2}\right)^{2}, \quad \epsilon>0 .
$$

Take the following dissipative force $\widehat{u}$ as a feedback control to the system $(\widehat{L}, \widehat{F}, \widehat{W})$ :

$$
\widehat{u}(x, \dot{x})=-c \dot{x}^{2} \mathbf{d} x^{2}, \quad c>0 .
$$

Then,

$$
\frac{d \widehat{E}}{d t}=-c\left(\dot{x}^{2}\right)^{2} \leq 0
$$

Hence, the equilibrium point $(0,0,0,0)$ is Lyapunov stable in the closed-loop dynamics $(\widehat{L}, \widehat{F}, \widehat{u})$.

We now show the asymptotic stability of the origin $(0,0,0,0)$ in the closed-loop system $(\widehat{L}, \widehat{F}, \widehat{u})$. Since $\widehat{E}$ has a strict local minimum at the origin and $d \widehat{E} / d t \leq 0$, there is a real number $l$ such that the set

$$
\Omega_{l}=\{(x, \dot{x}) \mid \widehat{E}(x, \dot{x}) \leq l\}
$$

is non-empty, compact and positively invariant. Define

$$
\begin{aligned}
\mathcal{E} & :=\left\{(x, \dot{x}) \in \Omega_{l} \mid d \widehat{E} / d t=0\right\} \\
& =\left\{\left(x^{1}, x^{2}, x^{1}, \dot{x}^{2}\right) \in \Omega_{l} \mid \dot{x}^{2}=0\right\}, \\
\mathcal{M} & :=\text { the largest invariant subset of } \mathcal{E} .
\end{aligned}
$$

Suppose that a trajectory

$$
(x(t), \dot{x}(t))=\left(x^{1}(t), x^{2}(t), \dot{x}^{1}(t), \dot{x}^{2}(t)\right)
$$

is contained in $\mathcal{M}$ for all $t \geq 0$. By the definition of $\mathcal{M}$, we have

$$
\begin{aligned}
\left(x^{1}(t), x^{2}(t), \dot{x}^{1}(t), \dot{x}^{2}(t)\right) & \\
& =\left(x^{1}(t), x^{2}(0), \dot{x}^{1}(t), 0\right)
\end{aligned}
$$

for all $t \geq 0$. Plugging (27) into the equations of motion of $(\widehat{L}, \widehat{F}, \widehat{u})$ yields

$$
\begin{aligned}
& \frac{-k_{1}\left(k_{2}-2 k_{1} \cos ^{2}\left(x^{1}\right)\right)}{k_{2}-k_{1} \cos ^{2}\left(x^{1}\right)} \ddot{x}^{1} \\
& =\frac{k_{1}^{2} k_{2} \cos \left(x^{1}\right) \sin \left(x^{1}\right)}{\left(k_{2}-k_{1} \cos ^{2}\left(x^{1}\right)\right)^{2}}\left(\dot{x}^{1}\right)^{2}-k_{1} \sin \left(x^{1}\right), \\
& -k_{1} \cos \left(x^{1}\right) \ddot{x}^{1}+k_{1} \sin \left(x^{1}\right)\left(\dot{x}^{1}\right)^{2}+\epsilon x^{2}(0) \\
& =-\frac{2 k_{1}^{2} \sin \left(x^{1}\right) \cos ^{2}\left(x^{1}\right)}{k_{2}-k_{1} \cos ^{2}\left(x^{1}\right)}\left(\dot{x}^{1}\right)^{2} .
\end{aligned}
$$


If $x^{1}(t)=0$ for all $t \geq 0,(29)$ implies that $x^{2}(0)=0$ for all $t \geq 0$. Hence, we will have $\left(x^{1}(t), x^{2}(t), \dot{x}^{1}(t), \dot{x}^{2}(t)\right)=(0,0,0,0)$ for all $t \geq$ 0 . Now, suppose that $x^{1}(t) \neq 0$ for some $t \geq$ 0 . Notice that the following quantity $C_{o}-$ it is the part of the energy $\widehat{E}$ corresponding to the variables $\left(x^{1}, \dot{x}^{1}\right)$, or it is the energy of $(28)-$ is constant along the trajectory of (28):

$$
C_{o}=-\frac{k_{1}\left(k_{2}-2 k_{1} \cos ^{2}\left(x^{1}\right)\right)}{2\left(k_{2}-k_{1} \cos ^{2}\left(x^{1}\right)\right)}\left(\dot{x}^{1}\right)^{2}-k_{1} \cos \left(x^{1}\right) \text {. }
$$

Notice that $C_{o}$ has a strict local minimum at $\left(x^{1}, \dot{x}^{1}\right)=(0,0)$. Hence, the curve $\left(x^{1}(t), \dot{x}^{1}(t)\right)$ will oscillate about $(0,0)$, so for all $t \geq 0$

$$
\min _{s \in[0, \infty)} x^{1}(s)=-a \leq x^{1}(t) \leq b=\max _{s \in[0, \infty)} x^{1}(s)
$$

for some $a, b>0$. Now, solve (28) for $\ddot{x}^{1}$, substitute it into (29), solve the resultant equation for $\left(\dot{x}^{1}\right)^{2}$, and then substitute it into (30). Then, along the trajectory $\left(x^{1}(t), x^{2}(0), \dot{x}^{1}(t), 0\right)$ the following holds:

$$
\begin{aligned}
C_{o} & =-k_{1} \cos \left(x^{1}\right) \\
& +\frac{\cos \left(x^{1}\right)\left(k_{1} \cos ^{2}\left(x^{1}\right)-k_{2}\right)\left(2 k_{1} \cos ^{2}\left(x_{1}\right)-k_{2}\right)}{2\left(2 k_{1}^{2} \cos ^{4}\left(x^{1}\right)-k_{2}^{2}\right)} \\
& -\frac{\epsilon x^{2}(0)\left(2 k_{1} \cos ^{2}\left(x^{1}\right)-k_{2}\right)^{2}}{2 \sin \left(x^{1}\right)\left(2 k_{1}^{2} \cos ^{4}\left(x^{1}\right)-k_{2}^{2}\right)} .
\end{aligned}
$$

By (31), the identity (32) must hold in the interval $[-a, b]$ of $x^{1}$ with $a, b>0$. Since only the term with the factor $\sin \left(x^{1}\right)$ in (32) is an odd function, that term must vanish (also notice only the term with the factor $\sin \left(x^{1}\right)$ blows up at 0 ). So, we have

$$
x^{2}(0)=0 \text {. }
$$

Then, the following must hold in the interval $[-a, b]$ of $x^{1}$ :

$$
\begin{aligned}
C_{o} & =-k_{1} \cos \left(x^{1}\right) \\
& +\frac{\cos \left(x^{1}\right)\left(k_{1} \cos ^{2}\left(x^{1}\right)-k_{2}\right)\left(2 k_{1} \cos ^{2}\left(x_{1}\right)-k_{2}\right)}{2\left(2 k_{1}^{2} \cos ^{4}\left(x^{1}\right)-k_{2}^{2}\right)}
\end{aligned}
$$

which is impossible unless $x^{1}(t)$ is constant. So far, we have shown that

$$
\left(x^{1}(t), x^{2}(t), \dot{x}^{1}(t), \dot{x}^{2}(t)\right)=\left(x^{1}(0), 0,0,0\right) .
$$

Substitution of this into (29) implies $\sin \left(x^{1}(0)\right)=$ 0 . It follows that $x^{1}(0)=0$. Therefore, the only possible trajectory in the set $\mathcal{M}$ is the equilibrium, $(0,0,0,0)$ only. By LaSalle's theorem, the origin is an asymptotically stable equilibrium in the closedloop system $(\widehat{L}, \widehat{F}, \widehat{W})$. The feedback control $u$ to the original system can be derived from (2). Notice that the gyroscopic force $\widehat{F}$ was useful in this example.

\section{REFERENCES}

Auckly, D. and L. Kapitanski (2003). On the $\lambda$ equations for matching control laws. SIAM J. Control Optim. 41(5), 1372-1388.
Auckly, D., L. Kapitanski and W. White (2000). Control of nonlinear underactuated systems. Comm. Pure Appl. Math. 53(3), 354-369.

Blankenstein, G., R. Ortega and A. J. VanDer Schaft (2002). The matching conditions of controlled Lagrangians and IDA-passivity based control. Int. J. Control 75(9), 645-665.

Bloch, A. M., D. E. Chang, N. E. Leonard and J. E. Marsden (2001). Controlled Lagrangians and the stabilization of mechanical systems II: Potential shaping. IEEE Trans. Automat. Control 46(10), 1556-1571.

Bloch, A. M., N. E. Leonard and J. E. Marsden (1997). Stabilization of mechanical systems using controlled Lagrangians. Proc. $C D C$ 36, 2356-2361.

Bloch, A. M., N. E. Leonard and J. E. Marsden (1999). Stabilization of the pendulum on a rotor arm by the method of controlled Lagrangians. Proc. of ICRA '99 pp. 500-505.

Bloch, A. M., N. E. Leonard and J. E. Marsden (2000). Controlled Lagrangians and the stabilization of mechanical systems I: The first matching theorem. IEEE Trans. Automat. Control 45(12), 2253-2270.

Chang, D. E. (2002). Controlled Lagrangian and Hamiltonian Systems. PhD thesis. California Institutde of Technology.

Chang, D. E., A. M. Bloch, N. E. Leonard, J. E. Marsden and C. A. Woolsey (2002). The equivalence of controlled Lagrangian and controlled Hamiltonian systems. ESAIM COCV 8, Sp. Iss., 393-422.

Chang, D. E. and J. E. Marsden (2004). Reduction of controlled Lagrangian and Hamiltonian systems with symmetry. SIAM J. Control Optim., To appear.

Hamberg, J. (2000). Controlled Lagrangians, symmetries and conditions for strong matching. In: Lagrangian and Hamiltonian Methods for Nonlinear Control : IFAC Workshop (N. E. Leonard and R. Ortega, Eds.). Pergamon. pp. $62-67$.

Ortega, R., M. W. Spong, F. Gomez-Estern and G. Blankenstein (2002). Stabilization of a class of undercactuated mechanical systems via interconnection and damping assignment. IEEE Trans. Automat. Control 47(8), 12181233.

Spivak, M. (1979). A Comprehensive Introduction to Differential Geometry. Vol. I. 2 ed.. Publish or Perish. Houston.

van der Schaft, A. (2000). L $L_{2}$-Gain and Passivity Techniques in Nonlinear Control. Communication \& Control Engineering Series. Springer-Verlag. 